\title{
Discussion of 'On the theory of standing waves in tyres at high vehicle speeds' by V.V. Krylov and O. Gilbert, Journal of Sound and Vibration 329 (2010) 4398-4408
}

\author{
W R Graham \\ University of Cambridge, Department of Engineering, Trumpington Street, Cambridge, CB2 1PZ
}

Keywords: tyre vibration, tyre standing waves, critical speed

\section{Introduction}

In Ref. [1], Krylov and Gilbert propose that the standing wave patterns observed in vehicle tyres above a certain, 'critical', speed can be interpreted in terms of a simplified model: an infinite, stationary beam subjected to a travelling point load. Two fundamental assumptions are implicit in this approach. First, the infinite beam must be accepted as a suitable representation of the ring-like tyre structure. Second, the change in reference frame must be legitimate. The purpose of this discussion is to assess these assumptions. It will be argued that the first is valid, at least qualitatively, but that the second is inadmissible due to the neglect of rotational inertia effects.

\section{Assessment}

To examine the first assumption, let us provisionally accept that the tyre structure can be modelled exactly as a one-dimensional beam, i.e. that the dispersion relation between the (radian) frequency, $\omega$, and free-wave speed, $c$, of harmonic displacements is the same for both. Then the question is simply whether the beam and the stationary tyre both exhibit an infinite response (in the absence of damping) to a point load travelling at a certain speed, and whether that speed is the same in each case. For the beam, it is given by the minimum value of the free-wave phase speed, $c(\omega)$. For the tyre, the condition is $[2]^{1}$

$$
\omega_{n}=n \Omega,
$$

in which $\omega_{n}$ is the resonance frequency of the mode at azimuthal order $n$, and $\Omega$ is the angular velocity of the load. This condition can be linked to the free-wave speed via the observation that the mode is a standing wave formed by the superposition of two free waves. Specifically, the dependence of the modal displacements on time, $t$, and angle, $\phi$, can be expressed, without loss of generality, as

\footnotetext{
${ }^{1}$ Note that, while Ref. [2] is presented as an analysis of a rotating tyre subject to a stationary load, rotational inertia effects are not included. Its results should therefore be viewed as applying instead to the stationary-tyre/rotating-load problem.
} 


$$
2 \cos \left(\omega_{n} t\right) \cos (n \phi) .
$$

This expression is the sum of the waveforms $\cos \left(\omega_{n} t-n \phi\right)$ and $\cos \left(\omega_{n} t+n \phi\right)$, which travel in opposite directions around the ring at speed $\omega_{n} R / n$ (where $R$ is the radius). Under the condition of Eq. (1) this is exactly equal to the load speed, $\Omega R$. The lowest load speed at which an infinite response occurs is thus again the minimum value of the free-wave speed ${ }^{2}$. Therefore any discrepancy between the critical-speed values for the beam and the tyre will be due solely to the discrete 'sampling' of the dispersion relation by the modes of the tyre. In reality, there will inevitably also be imperfections in the beam representation of the tyre dispersion relation; even if the material properties of the belt are perfectly described, the absence of curvature leads to noticeable discrepancies at low values of $n$. The first assumption will thus introduce some quantitative error; however it seems unlikely to lead to fundamental differences in behaviour between the model and the real system. In particular, one would still expect the speed-coincidence condition to give a reasonable estimate of the critical load speed.

The second assumption concerns the change in reference frame. So far, we have considered a stationary tyre with a moving load. In reality, the load is stationary (relative to the vehicle axle) and the tyre rotates. Since the transformation between these two configurations is not Galilean, the equations of motion now differ via additional, inertial terms, specifically those associated with the Coriolis and centripetal accelerations. For 'inextensible' rings, their influence can be described analytically (see, for example, Ref. [4] and the works cited therein). In the rotating frame, the free-wave speed of the component travelling in the same direction as the load is given by

$$
\frac{R}{n}\left[\sqrt{\omega_{n}^{2}+\frac{n^{2}\left(n^{2}-1\right)^{2}}{\left(n^{2}+1\right)^{2}} \Omega^{2}}+\frac{2 n}{n^{2}+1} \Omega\right] .
$$

It is straightforward to show that this expression is always greater than the load speed, $R \Omega$, for all admissible values of $n$ (recall that radial deformations with $n=0$ are not possible for an inextensible ring). In other words, the speed-coincidence condition invoked for both the beam and the stationary ring can never be met for the rotating ring. At the stationary-ring coincidence speed, the free-wave speed exceeds the load speed by a factor

$$
\left[\sqrt{1+\frac{\left(n^{2}-1\right)^{2}}{\left(n^{2}+1\right)^{2}}}+\frac{2}{n^{2}+1}\right]
$$

The restriction of (mean-plane) inextensibility is, of course, artificial, but is consistent with Krylov and Gilbert's beam model (which admits bending motions only). In any case, Huang and Soedel have confirmed that it is not crucial to the result $[5]^{3}$. The key point is that the behaviour of a rotating ring with relatively high in-plane stiffness, excited by a stationary force, differs fundamentally from that of the stationary ring driven by a rotating force. The assumption that

\footnotetext{
${ }^{2}$ This condition has also been noted by Metrikine and Tochilin [3], who analysed the same problem under high damping conditions.

${ }^{3}$ It should be noted in passing that Padovan's calculations [6], which apparently show resonant excitation of rotating ring waves by a stationary force, are in direct opposition to this finding. While there is insufficient information in Ref. [6] to allow definitive explanation of this conflict, it should be noted that Padovan employs incomplete governing equations. In this author's view, the results of Ref. [5] carry the greater authority.
} 
road-contact loading of a moving vehicle tyre is equivalent to travelling excitation of a stationary tyre is thus not tenable in modelling the critical-speed phenomenon.

It should, however, be recognised that Krylov and Gilbert are not alone in this error. Indeed, it is encouraged by the fact that other, apparently analogous, critical-speed phenomena can legitimately be explained by the speed-coincidence argument. The balloon-tyre experiments of Chatterjee et al. [7] are a case in point ${ }^{4}$. The critical speed of a rolling rigid cylinder with a thin viscoelastic coating is similarly associated with the speed of free longitudinal waves in the coating [8]. In fact, it appears that belted vehicle tyres are anomalous in having such a strong dependence of free-wave speed on rotation that coincidence is not possible. Presumably this arises because of their high in-plane, relative to bending, stiffness.

One possible response to the objections raised here is the observation that, when damping is included, significant proportions of the worst-case deformations are realised at load speeds some way below the (undamped) free-wave speed $[3,8]$. On this basis, one could argue that the inability to achieve coincident-speed forcing on a rotating belted tyre need not preclude the occurrence of visible standing-wave patterns. However, Huang and Soedel's forced-response results [5] provide little support for this hypothesis. Thus, it currently seems that more involved modelling will be necessary. An obvious candidate is Finite-Element-based computation. Unfortunately, the arbitrary-Lagrangian-Eulerian methods that represent the most efficient approach to steady rolling simulation are likely to suffer from lack-of-uniqueness problems at the extreme conditions of interest [9]. Meanwhile, time-resolved calculations starting from the unloaded, stationary state remain so numerically expensive that extremely high accelerations are necessary to reach the critical speed before the end of the simulation window [10], raising concerns that the solutions may be dominated by transients. Nonetheless, the results of Ref. [10] show promise, suggesting that this approach warrants further pursuit. It must, however, be supplemented with careful conceptual analysis if a workable theory of vehicle-tyre standing waves is to be developed.

\section{Conclusions}

This discussion has presented a critical assessment of an infinite-beam/travelling-load model for standing-wave generation in rotating vehicle tyres [1]. Its conclusions can be summarised as follows. The infinite one-dimensional beam subjected to a travelling point load is, qualitatively at least, equivalent to the excitation of a stationary tyre by a rotating load. The latter, however, differs crucially from the problem of interest, namely the excitation of a rotating, belted, tyre by a stationary load. For this reason, the approach proposed by Krylov and Gilbert appears unsuited to its intended purpose.

\section{References}

[1] V.V. Krylov, O. Gilbert, On the theory of standing waves in tyres at high vehicle speeds. Journal of Sound and Vibration 329 (2010) 4398-4408.

\footnotetext{
${ }^{4}$ Chatterjee et al.'s objection to a speed-coincidence explanation, on the basis that it does not account for the persistence of standing waves above the critical speed, is due to their use of an overly simplistic model dispersion relation, rather than any intrinsic problem with the theory. Both Soedel [2] and Metrikine and Tochilin [3] explicitly recognise the possibility of coincident excitation of free waves at load speeds above the critical value in their stationary ring analyses.
} 
[2] W. Soedel, On the dynamic response of rolling tyres according to thin shell approximations. Journal of Sound and Vibration 41 (1975) 233-246.

[3] A.V. Metrikine, M.V. Tochilin, Steady-state vibrations of an elastic ring under a moving load. Journal of Sound and Vibration 232 (2000) 511-524.

[4] M. Endo, K. Hatamura, M. Sakata, O. Taniguchi, Flexural vibration of a thin rotating ring. Journal of Sound and Vibration 92 (1984) 261-272.

[5] S.C. Huang, W. Soedel, Effects of Coriolis acceleration on the free and forced in-plane vibrations of rotating rings on elastic foundation. Journal of Sound and Vibration 115 (1987) 253-274.

[6] J. Padovan, On viscoelasticity and standing waves in tires. Tire Science and Technology 4 (1976), 233-246.

[7] A. Chatterjee, J.P. Cusumano, J.D. Zolock, On contact-induced standing waves in rotating tires: experiment and theory. Journal of Sound and Vibration 227 (1999) 1049-1081.

[8] X. Qiu, Full two-dimensional model for rolling resistance. II: Viscoelastic cylinders on rigid ground. Journal of Engineering Mechanics 135 (2009) 20-30.

[9] I. Stanciulescu, T.A. Laursen, On the interaction of frictional formulations with bifurcation phenomena in hyperelastic steady state rolling calculations. International Journal of Solids and Structures 43 (2006) 29592988.

[10] J.R. Cho, K.W. Kim, H.S. Jeong, Numerical investigation of tire standing wave using 3-D patterned tire model. Journal of Sound and Vibration 305 (2007) 795-807. 